\title{
FACTORIZATION OF CURVATURE OPERATORS
}

BY

\author{
JAAK VILMS
}

\begin{abstract}
Let $V$ be a real finite-dimensional vector space with inner product and let $R$ be a curvature operator, i.e., a symmetric linear map of the bivector space $\Lambda^{2} V$ into itself. Necessary and sufficient conditions are given for $R$ to admit factorization as $R=\Lambda^{2} L$, with $L$ a symmetric linear map of $V$ into itself. This yields a new characterization of Riemannian manifolds that admit local isometric embedding as hypersurfaces of Euclidean space.
\end{abstract}

1. Introduction. A curvature operator is a symmetric linear map $R: \Lambda^{2} V \rightarrow \Lambda^{2} V$, where $V$ is a real $n$-dimensional vector space with inner product and $\Lambda^{2} V$ is the associated $\left(\begin{array}{l}n \\ 2\end{array}\right)$-dimensional bivector space, supplied with the inner product induced from $V$. The problem solved in this paper is the following: to characterize those curvature operators $R: \Lambda^{2} V \rightarrow \Lambda^{2} V$ that can be factored as $R=\Lambda^{2} L$ for suitable symmetric linear maps $L: V \rightarrow V$.

This problem is closely related to the local embedding problem for Riemannian manifolds. Namely, if $M^{n}$ is an abstract Riemannian manifold, one asks for the least integer $p$ for which $M^{n}$ is locally isometrically embeddable into Euclidean space $E^{n+p}$. It is known that $p \leqslant \frac{1}{2} n(n+1)$ in the $C^{\infty}$ case, and that $p$ depends on the curvature tensor of $M^{n}$. At each point of $M^{n}$, this tensor can be represented as a symmetric linear map $R: \Lambda^{2} V \rightarrow \Lambda^{2} V$, where $V$ is the tangent space, endowed with the inner product given by the metric tensor. If $M^{n}$ is isometrically embeddable into $E^{n+p}$, then $R$ must at each point admit factorization as a sum $R=\Lambda^{2} L_{1}$ $+\cdots+\Lambda^{2} L_{p}$ for some symmetric linear maps $L_{i}: V \rightarrow V$. In general, it is not known how to determine the minimum-length factorization of $R$. The purpose of this paper is to characterize those $R$ which admit the shortest possible factorization, namely, $p=1$.

For this case, the factorization is in fact equivalent to local embeddability into $E^{n+1}$. Namely, T. Y. Thomas has shown [8] that an abstract Riemannian manifold $M^{n}$ with rank $R \geqslant 6$ admits local isometric embedding into $E^{n+1}$ if and only if $R$ can be factored as $R=\Lambda^{2} L$ at each point. Hence, the main result of the present paper provides necessary and sufficient conditions for local isometric embedding into $E^{n+1}$ when curvature has rank $\geqslant 6$.

The $p=1$ local embedding problem has been considered before by several authors, each working with quite different methods. Namely, T. Y. Thomas [8] obtained a solution by algebraic means, involving certain $3 \times 3$ determinants in the

Received by the editors January 18, 1979 and, in revised form, November 28, 1979.

1980 Mathematics Subject Classification. Primary 53B20; Secondary 53B25, $15 A 75$. 
matrix components of R. Rozenson [7] noted that the determinants in Thomas' solution were not coordinate-invariant; she obtained some rather complicated tensor expressions involving $R$, and gave a solution in terms of these tensors. Yanenko [11] gave essentially the same solution as Thomas, although he worked with differential 2-forms rather than just the matrix components of $R$.

The methods of this paper are different. The solution given here involves the properties of $R$ in relation both to the vector space structure and to the exterior product structure of the space $\Lambda^{2} V$. In fact, the principal condition on $R$ is that it must preserve the decomposability of bivectors. It is hoped that the methods used here will be useful also for studying the $p \geqslant 2$ case.

The main result of the paper is Theorem 7.8, contained in $\$ 7$ below.

The author wishes to thank, first, Professor Robert Liebler, for valuable references and for discussions about correlations and projective geometry, and second, the referee, for improving the proofs of Lemma 4.1 and Proposition 4.3.

2. Plan of work. Let $R: \Lambda^{2} V \rightarrow \Lambda^{2} V$ be a curvature operator. Our problem is to find conditions on $R$ that permit $R$ to be factored as $R=\Lambda^{2} L$, for a suitable linear map $L: V \rightarrow V$. This problem was solved in [9] for the case of nonsingular $R$, by means of a two-stage procedure. Namely, $R$ was first factored as $R= \pm \Lambda^{2} L$ and then the minus sign was removed. The results obtained in [9] can be applied to the case of an $R$ with nontrivial kernel as follows.

Since $R$ is symmetric, the vector space $\Lambda^{2} V$ admits the orthogonal decomposition $\Lambda^{2} V=\operatorname{ker} R+\operatorname{im} R$, and $R$ splits into the sum $R=R_{0}+R_{1}$, where $R_{1}: \operatorname{im} R \rightarrow$ im $R$ is a nonsingular linear map, and $R_{0}$ : $\operatorname{ker} R \rightarrow \operatorname{im} R$ is identically zero. If it happens that the subspace im $R$ of $\Lambda^{2} V$ is of the form $\Lambda^{2} U$ for some subspace $U$ of $V$, then the first result of [9] (cf. Proposition 7.1 below) can be used to factor the map $R_{1}$ as $R_{1}= \pm \Lambda^{2} L_{1}$, for a suitable linear map $L_{1}: U \rightarrow U$. The full factorization $R= \pm \Lambda^{2} L$ is then obtained by setting $L=L_{0}+L_{1}$, where $L_{0}: V \rightarrow U$ is the zero map. Finally, the minus sign can be removed by the same technique as in [9].

Therefore, the first task in this paper is to find conditions on $R$ that allow its image to be factored as im $R=\Lambda^{2} U$, for a suitable subspace $U$ of $V$.

We assume the elements of multilinear algebra (cf. [6], [10], and [4] especially for inner products).

3. Decomposability preserving maps and the subspace $U$. A linear map $R$ : $\Lambda^{2} V \rightarrow \Lambda^{2} V$ is said to preserve decomposability if each nonzero decomposable bivector either has a nonzero decomposable image or gets mapped to zero. Since we include zero in the set $G$ of decomposable bivectors, this happens precisely when $R(G) \subset G$.

Preservation of decomposability is the main condition on $R$ which is needed to factor the image of $R$ as im $R=\Lambda^{2} U$. In this section we give some equivalent formulations and general consequences of this condition, and we also define the subspace $U \subset V$. The proofs are omitted, since they are straightforward. We remind the reader that decomposable bivectors $\alpha, \beta$ are called adjacent if $\alpha \beta=0$. 
Proposition 3.1. (i) If $R$ preserves decomposability, then it also preserves adjacence.

(ii) $R$ preserves decomposability if and only if $R\left(x_{1} x_{2}\right) R\left(x_{3} x_{4}\right)=-R\left(x_{1} x_{3}\right) R\left(x_{2} x_{4}\right)$ for all $x_{1}, x_{2}, x_{3}, x_{4} \in V$.

(iii) $R$ preserves decomposability if and only if there exists a well-defined linear map $\Lambda^{2} R: \Lambda^{4} V \rightarrow \Lambda^{4} V$ satisfying the equation $\Lambda^{2} R(\alpha \beta)=R \alpha R \beta$ for all $\alpha, \beta \in \Lambda^{2} V$.

(iv) If $R$ preserves decomposability and is symmetric, then for all $\alpha, \beta \in \operatorname{im} R$, $\alpha \beta=0$ if and only if $R \alpha R \beta=0$.

Definition 3.2. Let $U \subset V$ be the subspace spanned by all planes $\{\omega\}$ belonging to decomposable bivectors $\omega \in \operatorname{im} R$.

Proposition 3.3. (i) If im $R$ is spanned by decomposable bivectors $\omega_{1}, \ldots, \omega_{N}$, then $U$ is spanned by the planes $\left\{\omega_{1}\right\}, \ldots,\left\{\omega_{N}\right\}$ of $V$, and $\operatorname{im} R \subset \Lambda^{2} U$.

(ii) If $R$ preserves decomposability, then im $R \subset \Lambda^{2} U$.

Definition 3.2 is suitable for defining $U$ only when $R$ preserves decomposability. We shall indicate some more general definitions for $U$, since these permit $\operatorname{dim} U$ to be related to other known numerical invariants of $R$. Define the absolute nullspace of a curvature operator $R$ to be the subspace $N$ in $V$ consisting of all vectors $x$ such that $R x y=0$ for all $y$ in $V$; define its "rank" subspace to be the smallest subspace $W$ in $V$ such that im $R \subset \Lambda^{2} W$. Then $\operatorname{dim} N$ is called the nullity index of $R$ (cf. [5, p. 347]), and $\operatorname{dim} W$ is called the "rank" of $R$ (cf. [11, $\$ 9$ on p. 34 and $\S 4$ on p. 76]). The codimension of $N$ is called the conullity or type of $R$. Clearly, it can also be described as the rank of the linear mapping $F: V \rightarrow \operatorname{Hom}\left(V, \Lambda^{2} V\right)$, where $F(x) y=$ Rxy (cf. [8, p. 186]).

Proposition 3.4. (i) $W=N^{\perp}$; hence "rank" $R=$ type $R$.

(ii) If $R$ preserves decomposability, then $U=W=N^{\perp}$; hence $\operatorname{dim} U=$ type $R$.

(iii) If $R= \pm \Lambda^{2} L$ for some linear map $L: V \rightarrow V$, then $U=\operatorname{im} L$; hence $\operatorname{dim} U=$ type $R=\operatorname{rank} L$.

4. Factoring $\operatorname{im} R$ when $\Lambda^{2} R \not 0$. Henceforth we assume $R$ is a decomposability-preserving curvature operator. In this section we obtain the factorization im $R=\Lambda^{2} U$ for the case $\Lambda^{2} R \neq 0$.

Any pair of decomposable bivectors $\alpha_{1}, \alpha_{2}$ with $\alpha_{1} \alpha_{2} \neq 0$ can be written as $\alpha_{1}=a_{1} a_{2}, \alpha_{2}=a_{3} a_{4}$, where $a_{1}, a_{2}, a_{3}, a_{4}$ are independent. If we suppose that $R \alpha_{1} R \alpha_{2} \neq 0$, then we can similarly write $R \alpha_{1}=b_{1} b_{2}, R \alpha_{2}=b_{3} b_{4}$, where $b_{1}, b_{2}, b_{3}, b_{4}$ are independent.

LEMMA 4.1. We may assume that

$$
\left.\begin{array}{rl}
R a_{1} a_{2} & =b_{1} b_{2}, \quad R a_{1} a_{3}=b_{1} b_{3} \\
R a_{2} a_{3} \\
R a_{1} a_{4}
\end{array}\right\}= \pm \begin{cases}b_{2} b_{3} & \text { or } \quad \pm\left\{\begin{array}{l}
b_{1} b_{4} \\
b_{1} b_{4} b_{3}
\end{array} \quad(\text { both }+ \text { or }-)\right. \\
R a_{2} a_{4} & =b_{2} b_{4}, \quad R a_{3} a_{4}=b_{3} b_{4}\end{cases}
$$


Proof. Consider the planes $\left\{R a_{i} a_{j}\right\}$ in $U$. Define vectors $b_{1}, b_{2}, b_{3}, b_{4}$ by the conditions

$$
\begin{array}{ll}
\left\{R a_{1} a_{2}\right\} \cap\left\{R a_{1} a_{3}\right\}=\left\{b_{1}\right\}, & \left\{R a_{1} a_{2}\right\} \cap\left\{R a_{2} a_{4}\right\}=\left\{b_{2}\right\}, \\
\left\{R a_{1} a_{3}\right\} \cap\left\{R a_{3} a_{4}\right\}=\left\{b_{3}\right\}, & \left\{R a_{2} a_{4}\right\} \cap\left\{R a_{3} a_{4}\right\}=\left\{b_{4}\right\} .
\end{array}
$$

Then these $b_{i}$ are independent and $R a_{1} a_{2}=x_{1} b_{1} b_{2}, R a_{3} a_{4}=x_{2} b_{3} b_{4}, R a_{1} a_{3}=$ $x_{3} b_{1} b_{3}, R a_{2} a_{4}=x_{4} b_{2} b_{4}$, where $x_{i}$ are nonzero scalars. Put $b_{1}^{\prime}=x_{3} b_{1}, b_{2}^{\prime}=x_{1} b_{2} / x_{3}$, $b_{3}^{\prime}=b_{3}, b_{4}^{\prime}=x_{2} b_{4}$; then $R a_{1} a_{2}=b_{1}^{\prime} b_{2}^{\prime}, R a_{3} a_{4}=b_{3}^{\prime} b_{4}^{\prime}$, and $R a_{2} a_{4}=y b_{2}^{\prime} b_{4}^{\prime}$. But now Proposition 3.1(ii) implies that $b_{1}^{\prime} b_{2}^{\prime} b_{3}^{\prime} b_{4}^{\prime}=-y b_{1}^{\prime} b_{3}^{\prime} b_{2}^{\prime} b_{4}^{\prime}$, whence $y=1$. Dropping the primes, we have the first two and the last two of the desired equations.

To get the remaining equations, set $\alpha=R a_{1} a_{4}, \beta=R a_{2} a_{3}$. Then Proposition 3.1(ii) implies $\alpha \beta=b_{1} b_{2} b_{3} b_{4}$, which in turn implies $\alpha, \beta \in \Lambda^{2}\left\{b_{1}, b_{2}, b_{3}, b_{4}\right\}$. Moreover, since $\alpha, \beta$ are both adjacent to the four bivectors $b_{1} b_{2}, b_{1} b_{3}, b_{2} b_{4}$ and $b_{3} b_{4}$, we must have

$$
\alpha=x b_{2} b_{3}+y b_{1} b_{4} \text { and } \beta=z b_{2} b_{3}+w b_{1} b_{4},
$$

where $x, y, z$ and $w$ are scalars. Since $\alpha \alpha=0$ and $\beta \beta=0$, and $\alpha \beta=b_{1} b_{2} b_{3} b_{4}$, it follows that $x y=0, z w=0$ and $x w+y z=1$. This implies that either $x=0$, $w=0, y z=1$, or $y=0, z=0, x w=1$. Therefore, these two cases hold.

Case (i). $\alpha=z b_{1} b_{4}, \beta=z^{-1} b_{2} b_{3}$ or

Case (ii). $\alpha=z b_{2} b_{3}, \beta=z^{-1} b_{1} b_{4}$.

For Case (i), set $b_{1}^{\prime}=|z|^{1 / 2} b_{1}, b_{2}^{\prime}=|z|^{-1 / 2} b_{2}, b_{3}^{\prime}=|z|^{-1 / 2} b_{3}, b_{4}^{\prime}=|z|^{1 / 2} b_{4}$. Then $b_{1}^{\prime} b_{2}^{\prime}=b_{1} b_{2}, \quad b_{1}^{\prime} b_{3}^{\prime}=b_{1} b_{3}, \quad b_{2}^{\prime} b_{4}^{\prime}=b_{2} b_{4}, \quad b_{3}^{\prime} b_{4}^{\prime}=b_{3} b_{4}$. However, $b_{2}^{\prime} b_{3}^{\prime}=|z|^{-1} b_{2} b_{3}$ $= \pm z^{-1} b_{2} b_{3}= \pm \beta= \pm R a_{2} a_{3}$, and $b_{1}^{\prime} b_{4}^{\prime}=|z| b_{1} b_{4}= \pm \alpha= \pm R a_{1} a_{4}$, where both signs are + when $z>0$ and both are - when $z<0$.

For Case (ii) set $b_{1}^{\prime}=|z|^{-1 / 2} b_{1}, b_{2}^{\prime}=|z|^{1 / 2} b_{2}, b_{3}^{\prime}=|z|^{1 / 2} b_{3}, b_{4}^{\prime}=|z|^{-1 / 2} b_{4}$. Then $b_{1}^{\prime} b_{2}^{\prime}=b_{1} b_{2}, b_{1}^{\prime} b_{3}^{\prime}=b_{1} b_{3}, b_{2}^{\prime} b_{4}^{\prime}=b_{2} b_{4}$ and $b_{3}^{\prime} b_{4}^{\prime}=b_{3} b_{4}$. However, $b_{2}^{\prime} b_{3}^{\prime}=|z| b_{2} b_{3}$ $= \pm z b_{2} b_{3}= \pm \alpha= \pm R a_{1} a_{4}$, and $b_{1}^{\prime} b_{4}^{\prime}=|z|^{-1} b_{1} b_{4}= \pm z^{-1} b_{1} b_{4}= \pm \beta= \pm R a_{2} a_{3}$, with the same type of sign. Q.E.D.

LEMMA 4.2. Let $\omega_{1}$ and $\omega_{2}$ be decomposable bivectors in im $R$, such that $\omega_{1} \omega_{2} \neq 0$. Then the 4-dimensional subspace $U_{1}=\left\{\omega_{1}\right\}+\left\{\omega_{2}\right\}$ of $V$ has the property that $\Lambda^{2} U_{1} \subset \operatorname{im} R$.

Proof. Let $\omega_{1}=R \alpha_{1}$ and $\omega_{2}=R \alpha_{2}$. By the orthogonal decomposition of $\Lambda^{2} V$ given in $\$ 2$, it may be assumed that $\alpha_{1}$ and $\alpha_{2}$ are in im $R$. But then Proposition 3.1(iv) implies that both $\alpha_{1}$ and $\alpha_{2}$ are decomposable and that $\alpha_{1} \alpha_{2} \neq 0$. Hence $\alpha_{1}$, $\alpha_{2}$ can be expressed as $\alpha_{1}=a_{1} a_{2}, \alpha_{2}=a_{3} a_{4}$. Let $\omega_{1}=b_{1} b_{2}$ and $\omega_{2}=b_{3} b_{4}$. Then $\left\{b_{1}, b_{2}, b_{3}, b_{4}\right\}+\left\{b_{1} b_{2}\right\}=\left\{b_{3} b_{4}\right\}=\left\{\omega_{1}\right\}+\left\{\omega_{2}\right\}=U_{1}$. Now Lemma 4.1 implies that each of the bivectors $b_{1} b_{2}, \ldots, b_{3} b_{4}$ in the basis of $\Lambda^{2} U_{1}$ is an image by $R$ of a bivector in $\Lambda^{2} V$; hence $\Lambda^{2} U_{1}$ must be contained in im $R$. Q.E.D.

Proposition 4.3. If $R: \Lambda^{2} V \rightarrow \Lambda^{2} V$ is a curvature operator which preserves decomposability, and if $\Lambda^{2} R \neq 0$, then $\operatorname{im} R=\Lambda^{2} U$. Moreover, $\operatorname{dim} U \geqslant 4$ and rank $R \geqslant 6$. 
Proof. Let $U$ be defined by Definition 3.2. Then by Proposition 3.3(ii), im $R \subset$ $\Lambda^{2} U$, and it suffices to prove that $\Lambda^{2} R \neq 0$ implies $\Lambda^{2} U \subset$ im $R$. To establish this inclusion, it is enough to show that each decomposable bivector of $\Lambda^{2} U$ is in im $R$, i.e., that $x, y \in U$ implies $x y \in \operatorname{im} R$.

Let $x, y \in U$. By definition of $U$, there exist decomposable bivectors $\omega_{1}, \omega_{2} \in$ im $R$ such that $x \in\left\{\omega_{1}\right\}, y \in\left\{\omega_{2}\right\}$.

If $\omega_{1} \omega_{2} \neq 0$, then Lemma 4.2 implies that $\Lambda^{2} U_{1} \subset \operatorname{im} R$, where $U_{1}=\left\{\omega_{1}\right\}+$ $\left\{\omega_{2}\right\}$. But both $x$ and $y$ are in $U_{1}$, so that $x y$ is in $\Lambda^{2} U_{1}$; hence $x y \in \operatorname{im} R$.

If $\omega_{1} \omega_{2}=0$, then we have $\omega_{1}=x z, \omega_{2}=z y$ for a suitable vector $z$, and we may assume $x, y, z$ independent. In this case Lemma 4.2 cannot be applied directly, so an auxiliary subspace must be used from which to get decomposable vectors of im $R$ that are not adjacent to $\omega_{1}$ or $\omega_{2}$. For this purpose, let $e_{1}, \ldots, e_{n}$ be a basis of $V$. Then Proposition 3.1(iii) implies that if $\Lambda^{2} R \neq 0$ then not all $R e_{i} e_{j} R e_{k} e_{l}$ with $i<j<k<l$ can vanish. By relabeling the basis, it may be assumed that $R e_{1} e_{2} R e_{3} e_{4} \neq 0$. Then Lemma 4.2 implies that $\Lambda^{2} U_{0} \subset \operatorname{im} R$, where $U_{0}=\left\{\operatorname{Re}_{1} e_{2}\right\}$ $+\left\{R e_{3} e_{4}\right\}$. Let us consider the relative positions of the 4-dimensional space $U_{0}$ and the 3-dimensional space $W_{0}=\left\{\omega_{1}\right\}+\left\{\omega_{2}\right\}=\{x, y, z\}$.

If $W_{0} \subset U_{0}$, then $x, y \in U_{0}$, so $x y \in \Lambda^{2} U_{0}$, whence $x y \in \operatorname{im} R$, and we are done. On the other hand, if $W_{0} \nsubseteq U_{0}$, then $U_{0} \cap W_{0}$ has dimension $\leqslant 2$. Hence $\left(U_{0} \cap W_{0}\right)^{\perp} \cap U_{0}$ has dimension $\geqslant 2$ and therefore contains a pair of independent vectors $v_{1}, v_{2}$. Setting $\omega_{3}=v_{1} v_{2}$, we have $\omega_{3} \in \operatorname{im} R, \omega_{2} \omega_{3} \neq 0$ and $z, y, v_{1}, v_{2}$ independent. Now we can apply Lemma 4.2 to $\omega_{2}, \omega_{3}$ to conclude $\Lambda^{2} U_{1} \subset$ im $R$, where $U_{1}=\left\{\omega_{2}\right\}+\left\{\omega_{3}\right\}$.

If $\left\{\omega_{1}\right\} \subset U_{1}$, then $x, y \in U_{1}$, whence $x y \in \operatorname{im} R$, and we are done. If $\left\{\omega_{1}\right\} \nsubseteq$ $U_{1}$, put $\omega_{4}=y v_{2}$; then $\omega_{1} \omega_{4} \neq 0$, because $\omega_{1} \omega_{4}=0$ would imply $\left\{\omega_{1}\right\} \subset U_{1}$. We again apply Lemma 4.2 to $\omega_{1}, \omega_{4}$, to conclude $\Lambda^{2} U_{2} \subset$ im $R$, where $U_{2}=\left\{\omega_{1}\right\}+$ $\left\{\omega_{4}\right\}=\left\{x, y, z, v_{2}\right\}$. Thus $x y \in \operatorname{im} R$. Q.E.D.

5. Correlations. The special case $\Lambda^{2} R \not 0$, rank $R=6$ presents an anomalous feature-the possibility of $R$ being a correlation-which must be excluded later. Therefore we include here a discussion of this case.

It follows from Proposition 4.3 that im $R=\Lambda^{2} U$ and $\operatorname{dim} U=4$. Hence the nonsingular map $R \lim R: \Lambda^{2} U \rightarrow \Lambda^{2} U$ satisfies the equations of Lemma 4.1, where $a_{1}, a_{2}, a_{3}, a_{4}$ and $b_{1}, b_{2}, b_{3}, b_{4}$ are now two bases of $U$. If $R a_{2} a_{3}= \pm b_{2} b_{3}$ and $R a_{1} a_{4}= \pm b_{1} b_{4}, R$ is called a collineation, whereas if $R a_{2} a_{3}= \pm b_{1} b_{4}$ and $R a_{1} a_{4}$ $= \pm b_{2} b_{3}, R$ is a correlation.

If our $R$ has the form $R= \pm \Lambda^{2} L$, then clearly it is a collineation. Hence for the case $\Lambda^{2} R \neq 0$, rank $R=6$, the condition that $R$ is not a correlation is necessary for $R$ to admit factorization as $R= \pm \Lambda^{2} L$.

The following three results give criteria for deciding whether $R$ is a collineation or a correlation.

Propositron 5.1. Let $R: \Lambda^{2} V \rightarrow \Lambda^{2} V$ be a decomposability-preserving curvature operator having rank 6. Let $e_{1}, \ldots, e_{n}$ be a basis of $V$ such that $e_{1}, \ldots, e_{4}$ is a basis of $U$. If $R e_{1} e_{2}=b_{1} b_{2}, R e_{1} e_{3}=b_{1} b_{3}, R e_{2} e_{3}= \pm b_{2} b_{3}$, then $R$ is a collineation, 
whereas if $R e_{2} e_{3}= \pm b_{1} b_{4}$, then $R$ is a correlation. (The vectors $b_{1}, \ldots, b_{4}$ are $a$ suitable independent set in $U$.)

Proof. Clear.

PROPOSITION 5.2. $R$ is a collineation or a correlation according to whether $\left\{R a_{1} a_{2}\right\}$ $+\left\{R a_{1} a_{3}\right\}+\left\{R a_{2} a_{3}\right\}$ is a 3- or 4-dimensional subspace of $V$, respectively, where $a_{1}, a_{2}, a_{3}$ is an arbitrary independent set in $V$.

Proof. Consider the mapping of planes of $V$ into planes of $V$ defined by $\{x y\} \rightarrow\{R x y\}$. If $R$ is a collineation, then the set of planes containing a given line gets mapped onto a set of planes that also contain a common line, and the set of planes contained in a given 3-space gets mapped onto a set of the same type. But if $R$ is a correlation, these types of sets of planes get reversed (cf. [3, p. 107]). Q.E.D.

Proposition 5.3. Let $R: \Lambda^{2} V \rightarrow \Lambda^{2} V$ be a decomposability-preserving curvature operator having rank 6. Let $e_{1}, \ldots, e_{n}$ be a basis of $V$ such that $e_{1}, e_{2}, e_{3}, e_{4}$ is a basis of $U$, and let $R_{k l}^{i j}$ be the matrix of $R$ with respect to the basis $e_{i} e_{j}, i<j$, of $\Lambda^{2} V$. Then $R$ is a collineation if and only if the following matrix has rank 4.

$$
\left(\begin{array}{cccc}
R_{12}^{23} & -R_{12}^{13} & R_{12}^{12} & 0 \\
R_{12}^{24} & -R_{12}^{14} & 0 & R_{12}^{12} \\
R_{13}^{23} & -R_{13}^{13} & R_{13}^{12} & 0 \\
R_{13}^{24} & -R_{13}^{14} & 0 & R_{13}^{12} \\
R_{23}^{23} & -R_{23}^{13} & R_{23}^{12} & 0 \\
R_{23}^{24} & -R_{23}^{14} & 0 & R_{23}^{12}
\end{array}\right)
$$

Proof. For a decomposable bivector $\alpha$ in $\Lambda^{2} U$ the plane $\{\alpha\}$ spanned by it in $V$ is given by

$$
\{\alpha\}=\{x \mid \alpha x=0\}=\left\{x \mid x^{i} \alpha^{j k}-x^{j} \alpha^{i k}+x^{k} \alpha^{i j}=0 \text { for } 1 \leqslant i<j<k \leqslant 4\right\},
$$

where $\alpha=\sum \alpha^{i j} e_{i} e_{j}$ with $1 \leqslant i<j \leqslant 4$. Since $\alpha$ is decomposable, $\alpha \alpha=0$, which gives $\alpha^{12} \alpha^{34}-\alpha^{13} \alpha^{24}+\alpha^{14} \alpha^{23}=0$. From this equation it can be seen that the four equations describing $\{\alpha\}$ are dependent, and that those two with indices $i, j, k$ equal to $1,2,3$ and $1,2,4$ are independent. Hence, as a subset of $\mathbf{R}^{4},\{\alpha\}$ is the nullspace of the matrix

$$
\left(\begin{array}{cccc}
\alpha^{23} & -\alpha^{13} & \alpha^{12} & 0 \\
\alpha^{24} & -\alpha^{14} & 0 & \alpha^{12}
\end{array}\right)
$$

If $\alpha=R e_{1} e_{2}$, then the corresponding matrix is given by the first two rows of the $6 \times 4$ matrix in the statement of this proposition; its nullspace is precisely $\left\{\operatorname{Re}_{1} e_{2}\right\}$, considered as a subspace of $\mathbf{R}^{4}$. The next two pairs of rows of that matrix correspond to $\left\{\operatorname{Re}_{1} e_{3}\right\}$ and $\left\{\operatorname{Re}_{2} e_{3}\right\}$. Therefore, the nullspace of the whole $6 \times 4$ matrix corresponds to the space $\left\{R e_{1} e_{2}\right\} \cap\left\{R e_{1} e_{3}\right\} \cap\left\{R e_{2} e_{3}\right\}$. Now $R$ is a collineation if and only if this space is $\{0\}$, which occurs exactly when the $6 \times 4$ matrix has maximal rank. Q.E.D. 
6. The case $\Lambda^{2} R \equiv 0$. It is easy to see from Proposition 3.1 that $\Lambda^{2} R \equiv 0$ if and only if $R\left(x_{1} x_{2}\right) R\left(x_{3} x_{4}\right)=0$ for all $x_{1}, x_{2}, x_{3}, x_{4}$ in $V$. This is a rather severe restriction on $R$; as a consequence, we can perform the factorings im $R=\Lambda^{2} U$ and $R= \pm \Lambda^{2} L$ both at the same time.

Proposition 6.1. Let $R: \Lambda^{2} V \rightarrow \Lambda^{2} V$ be a curvature operator which preserves decomposability and satisfies $\Lambda^{2} R \equiv 0$. Then $R= \pm \Lambda^{2} L$ for a suitable linear map $L$ : $V \rightarrow V$ if and only if rank $R=0$ or 1 , or rank $R=$ type $R=3$. Moreover, in case rank $R=0$ or 1 , the + sign may be assumed to hold, with $L$ symmetric.

Proof. Let $a_{1}, \ldots, a_{k}$ be a basis of $U$, with $U$ as in Definition 3.2. Since $R$ preserves decomposability, Proposition 3.3 states that $\operatorname{im} R \subset \Lambda^{2} U$. Hence $R$ restricts to a linear map $\Lambda^{2} U \rightarrow \Lambda^{2} U$ which still has the same set as image. Therefore the bivectors $R a_{i} a_{j}$ for $1 \leqslant i<j \leqslant k$ span im $R$, and $U=\sum\left\{R a_{i} a_{j}\right\}$ accordingly. Since $\Lambda^{2} R \equiv 0$, all the nonzero bivectors among these $R a_{i} a_{j}$ must be adjacent to each other, so that all the corresponding planes $\left\{R a_{i} a_{j}\right\}$ have 1-dimensional intersections. But then the result of $[1$, p. 16] implies that either (i) all of them contain one 1-dimensional subspace of $V$ or (ii) they are all contained in one 3-dimensional subspace of $V$.

In case (i), $R a_{i} a_{j}=b_{1} b_{i j}$ for all $1 \leqslant i<j \leqslant k$, where $b_{1}$ is a fixed vector in $U$, and $b_{i j}$ is taken equal to $b_{1}$ when $R a_{i} a_{j}=0$. By definition, $U$ is spanned by the planes $\left\{R a_{i} a_{j}\right\}=\left\{b_{1}, b_{i j}\right\}$ corresponding to the nonzero $R a_{i} a_{j}$. This means that these vectors $b_{1}, \ldots, b_{i j}, \ldots$ span $U$, which implies that $k$ of them are independent, including $b_{1}$. Denote these vectors by $b_{1}, b_{2}, \ldots, b_{k}$; they are a basis of $U$, and consequently the $k-1$ bivectors $b_{1} b_{2}, \ldots, b_{1} b_{k}$ are a basis of im $R$. Therefore $R$ has rank $k-1$.

In case (ii), all the planes $\left\{R a_{i} a_{j}\right\}$ lie in one 3-dimensional subspace of $V$, whence $U$ has dimension $k \leqslant 3$.

Now suppose $R= \pm \Lambda^{2} L$ for some linear map $L: V \rightarrow V$. It follows from Proposition 3.4 that $U=\operatorname{im} L$ and $\Lambda^{2} U=\operatorname{im} R$. This implies that rank $R=$ $\operatorname{dim} \Lambda^{2} U=\left(\begin{array}{l}k \\ 2\end{array}\right)$. In Case (i), this means $\left(\begin{array}{l}k \\ 2\end{array}\right)=k-1$, which implies $k=1$ or 2 , so that rank $R=0$ or 1 . In Case (ii), $k \leqslant 3$, i.e., $k=0,1,2$ or 3. Therefore, rank $R=\left(\begin{array}{l}k \\ 2\end{array}\right)=0,1$, or 3 . Hence $R= \pm \Lambda^{2} L$ implies the rank and dimension conditions in the proposition, noting that by Proposition 3.4(ii), $\operatorname{dim} U=$ type $R$.

To prove the converse, start with rank $R=0$. Then $R \equiv 0$, so $R=\Lambda^{2} L$ for $L \equiv 0$. Next, suppose rank $R=1$. This implies that $R e_{i} e_{j} \neq 0$ for some $i<j$, where $e_{1}, \ldots, e_{n}$ is any basis of $V$. This nonzero $R e_{i} e_{j}$ must be decomposable, since $R$ preserves decomposability; it also must span im $R$. Let $v_{1}, v_{2}$ be an orthonormal basis of the corresponding plane $\left\{R e_{i} e_{j}\right\}$. Therefore $v_{1} v_{2}$ also spans im $R$; in fact, im $R=\Lambda^{2} U$, where $U=\left\{v_{1}, v_{2}\right\}$. Since $R \mid$ im $R$ is nonsingular, there is a scalar $c \neq 0$ such that $R v_{1} v_{2}=c v_{1} v_{2}$. Extend $v_{1}, v_{2}$ to an orthonormal basis $v_{1}, v_{2}, v_{3}, \ldots, v_{n}$ of $V$; then $v_{1} v_{2}, v_{1} v_{3}, \ldots, v_{n-1} v_{n}$ is an orthonormal basis of $\Lambda^{2} V$. Since $R$ is symmetric, $\Lambda^{2} V$ splits into the orthogonal sum $\Lambda^{2} V=\operatorname{ker} R+\operatorname{im} R$. This implies that $v_{1} v_{3}, \ldots, v_{n-1} v_{n}$ is a basis of $\operatorname{ker} R$, so that $R v_{i} v_{j}=0$ when 
$(i, j) \neq(1,2)$. Now set $L v_{1}=c v_{1}, L v_{2}=v_{2}, L v_{i}=0$ for $3 \leqslant i$. Then $R=\Lambda^{2} L$. Note that $L$ is symmetric, and that rank $R=1$ implies $\Lambda^{2} R \equiv 0$.

Finally, suppose that rank $R=$ type $R=3$, i.e., $\operatorname{dim} U=3$. By the first part of this proof, three of the bivectors $R a_{i} a_{j}$ for $1 \leqslant i<j \leqslant k$, are independent and their planes span $U$. Now either Case (i) or Case (ii) holds.

In Case (i), it was shown above that $U$ has a basis $b_{1}, \ldots, b_{k}$ and that the bivectors $b_{1} b_{2}, \ldots, b_{1} b_{k}$ are a basis of im $R$. Since rank $R=3$, this basis contains exactly 3 bivectors, which implies $k=4$. But this contradicts the hypothesis that $\operatorname{dim} U=3$, which rules out this case.

In Case (ii), the planes $\left\{R a_{i} a_{j}\right\}$ are all contained in a 3-dimensional subspace of $V$, which implies $\operatorname{dim} U \leqslant 3$, so that also $\operatorname{dim} \Lambda^{2} U \leqslant 3$. Since by Proposition 3.3 im $R \subset \Lambda^{2} U$ and, by hypothesis, rank $R=3$, we have im $R=\Lambda^{3} U$ and $\operatorname{dim} U=$ 3. Let $v_{1}, v_{2}, v_{3}$ be an orthonormal basis of $U$. Then $v_{1} v_{2}, v_{1} v_{3}, v_{2} v_{3}$ is an orthonormal basis of $\Lambda^{2} U=\operatorname{im} R$ and the planes $\left\{R v_{1} v_{2}\right\},\left\{R v_{1} v_{3}\right\},\left\{R v_{2} v_{3}\right\}$ span $U$.

Consequently, these planes intersect each other in three distinct lines $\left\{b_{1}\right\},\left\{b_{2}\right\}$, $\left\{b_{3}\right\}$, where the vectors $b_{1}, b_{2}, b_{3}$ can be chosen so that $R v_{1} v_{2}=b_{1} b_{2}, R v_{1} v_{3}=b_{1} b_{3}$, $R v_{2} v_{3}=c b_{2} b_{3}$, with $c$ a nonzero scalar. It is not hard to see that the lengths and directions of the vectors $b_{1}, b_{2}, b_{3}$ can be adjusted to get $R v_{1} v_{2}=b_{1} b_{2}, R v_{1} v_{3}=$ $b_{1} b_{3}, R v_{2} v_{3}= \pm b_{2} b_{3}$. Now extend the basis $v_{1}, v_{2}, v_{3}$ to an orthonormal basis $v_{1}, v_{2}$, $v_{3}, v_{4}, \ldots, v_{n}$ of $V$. Then $v_{1} v_{2}, v_{1} v_{3} v_{1} v_{4}, \ldots, v_{n-1} v_{n}$ is an orthonormal basis of $\Lambda^{2} V$. Just as in the case of rank $R=1$ above, it follows from the symmetry of $R$ that $R v_{i} v_{j}=0$ when $(i, j)$ is not one of the pairs $(1,2),(1,3),(2,3)$.

Set $L v_{1}= \pm b_{1}, L v_{2}=b_{2}, L v_{3}=b_{3}$ and $L v_{i}=0$ for $4 \leqslant i$. Then $R= \pm \Lambda^{2} L$. Q.E.D.

7. The main factorization theorems. Here we bring together the results of the previous sections to obtain first $R= \pm \Lambda^{2} L$ and then $R=\Lambda^{2} L$. As indicated in $\S 2$, we shall use the results of [9] in a formulation extended to include the case $\operatorname{dim} V=4$.

Proposition 7.1. A nonsingular curvature operator $R: \Lambda^{2} V \rightarrow \Lambda^{2} V$, with $\operatorname{dim} V \geqslant$ 4, can be factored as $R= \pm \Lambda^{2} L$, for a suitable linear map $L: V \rightarrow V$, if and only if $R$ preserves decomposability and is not a correlation in the case $\operatorname{dim} V=4$.

Proof. This is just [9, Theorem 1], extended to include $\operatorname{dim} V=4$ (cf. [2, p. 38]). By Proposition 5.1, maps of form $R= \pm \Lambda^{2}$ are collineations, not correlations. Q.E.D.

Define two scalar invariants of $R$ as follows (cf. [9, p. 201]). Set

$$
\phi(R)=R_{k l}^{i j} R_{i q}^{k p} R_{j p}^{l q}, \quad \psi(R)=R_{k l}^{i j} R_{p q}^{k l} R_{i j}^{p q}
$$

where summation is understood over repeated indices, and $R_{k l}^{i j}$ is the matrix of $R$ with respect to any basis $e_{i} e_{j}, i<j$, corresponding to a basis $e_{1}, \ldots, e_{n}$ of $V$. 
Proposition 7.2 [9, TheOREM 3]. Suppose $R$ is a nonsingular curvature operator such that $R= \pm \Lambda^{2} L$ for a symmetric linear map $L: V \rightarrow V$, with $\operatorname{dim} V \geqslant 3$. Then $R=\Lambda^{2} L$ if and only if $\phi(R)+\frac{1}{4} \psi(R)>0$. Moreover, in case $n \equiv 3(\bmod 4)$, this inequality may be replaced by $\operatorname{det} R>0$.

THEOREM 7.3. Let $R: \Lambda^{2} V \rightarrow \Lambda^{2} V$ be a curvature operator. Then $R= \pm \Lambda^{2} L$ for some linear map $L: V \rightarrow V$ if and only if $R$ preserves decomposability and satisfies one of the following additional conditions.

(i) $\Lambda^{2} R \neq 0$ and $R$ is not a correlation in case rank $R=6$.

(ii) $\Lambda^{2} R \equiv 0$ and either rank $R=0$ or 1 , or rank $R=$ type $R=3$.

Proof. Suppose $\Lambda^{2} R \not 0$. If $R$ preserves decomposability, then by Proposition 4.3, rank $R \geqslant 6$ and $\operatorname{im} R=\Lambda^{2} U$, with $\operatorname{dim} U \geqslant 4$. If $R$ is nonsingular, then $U=V$. If $R$ is not a correlation, then $R= \pm \Lambda^{2} L$ follows from Proposition 7.1. If $R$ is singular, then by $\S 2, R_{1}=R \mid \operatorname{im} R: \Lambda^{2} U \rightarrow \Lambda^{2} U$ is a nonsingular symmetric linear map. By Proposition 7.1, it factors as $R_{1}= \pm \Lambda^{2} L_{1}$, provided $R_{1}$ is not a correlation (note that $R$ is a correlation if and only if $R_{1}$ is one). Therefore $\$ 2$ gives $R= \pm \Lambda^{2} L$, for a linear $L: V \rightarrow V$. Thus if $R$ preserves decomposability and satisfies (i), then $R= \pm \Lambda^{2} L$.

Suppose now that $\Lambda^{2} R \equiv 0$ and let $R$ preserve decomposability. Then Proposition 6.1 shows that (ii) implies $R= \pm \Lambda^{2} L$.

Conversely, assume $R= \pm \Lambda^{2} L$; clearly $R$ preserves decomposability. If $\Lambda^{2} R \equiv$ 0 , then Proposition 6.1 implies statement (ii). If $\Lambda^{2} R \neq 0$ and rank $R=6$, then Proposition 5.1 implies that $R$ is not a correlation. Q.E.D.

COROLlaRY 7.4. If $R: \Lambda^{2} V \rightarrow \Lambda^{2} V$ is a curvature operator that preserves decomposability and has rank $>6$, then it admits factorization as $R= \pm \Lambda^{2} L$ if and only if $\Lambda^{2} R \neq 0$.

In order to state the next theorem, we recall the Bianchi identity of Riemannian geometry. $R$ is said to satisfy the Bianchi identity if

$$
\left\langle R x_{1} x_{2}, x_{3} x_{4}\right\rangle+\left\langle R x_{2} x_{3}, x_{1} x_{4}\right\rangle+\left\langle R x_{3} x_{1}, x_{2} x_{4}\right\rangle=0
$$

for all $x_{1}, x_{2}, x_{3}, x_{4}$ in $V$.

THEOREM 7.5. Suppose $R$ is a curvature operator such that $R= \pm \Lambda^{2} L$ for a linear map $L: V \rightarrow V$ with rank $L \geqslant 3$. Then $L$ is symmetric if and only if $R$ satisfies the Bianchi identity.

Proof. Substituting $R= \pm \Lambda^{2} L$ into the left side of the Bianchi identity gives

$$
\begin{aligned}
\left\langle L x_{3}, x_{4}\right\rangle\left(\left\langle L x_{2}, x_{1}\right\rangle-\left\langle L x_{1}, x_{2}\right\rangle\right) & +\left\langle L x_{2}, x_{4}\right\rangle\left(\left\langle L x_{1}, x_{3}\right\rangle-\left\langle L x_{3}, x_{1}\right\rangle\right) \\
& +\left\langle L x_{1}, x_{4}\right\rangle\left(\left\langle L x_{3}, x_{2}\right\rangle-\left\langle L x_{2}, x_{3}\right\rangle\right) .
\end{aligned}
$$

If $L$ is symmetric, this clearly reduces to zero. Conversely, if the Bianchi identity holds, this expression is identically zero. Consider any two vectors $x_{1}, x_{2}$. It was observed in the proof of Proposition 6.1 that $R= \pm \Lambda^{2} L$ implies $U=\operatorname{im} L$ and $\Lambda^{2} U=\operatorname{im} R$. Hence, rank $R=\left(\begin{array}{c}k \\ 2\end{array}\right)$, where $k=\operatorname{dim} U$. Consequently, the hypothesis, rank $L \geqslant 3$, implies that $\operatorname{dim} U \geqslant 3$. Therefore the vectors $L x_{1}, L x_{2}$ are in $U$ 
and do not span all of $U$. Hence there exists a nonzero vector $x_{4} \in U$ such that $x_{4} \perp$ both $L x_{1}$ and $L x_{2}$. This gives the equation

$$
\left\langle L x_{3}, x_{4}\right\rangle\left(\left\langle L x_{2}, x_{1}\right\rangle-\left\langle L x_{1}, x_{2}\right\rangle\right)=0 .
$$

Now the map $L_{1}^{-1}=(L \mid U)^{-1}: U \rightarrow U$ is well defined. Set $x_{3}=L_{1}^{-1} x_{4}$; then $L x_{3}=L_{1} x_{3}=x_{4}$ and consequently $\left\langle L x_{3}, x_{4}\right\rangle=\left\langle x_{4}, x_{4}\right\rangle \neq 0$, since $x_{4}$ was chosen nonzero. Therefore, the term in parentheses in the above equation vanishes, whence $L$ is symmetric. Q.E.D.

THEOREM 7.6. Suppose $R$ is a curvature operator such that $R= \pm \Lambda^{2} L$ for $a$ symmetric linear map $L: V \rightarrow V$ having rank $L \geqslant 3$. Then $R=\Lambda^{2} L$ if and only if $\phi(R)+\frac{1}{4} \psi(R)>0$. Moreover, in case $n \equiv 3(\bmod 4)$, this inequality may be replaced by $\operatorname{det}(R \mid \operatorname{im} R)>0$.

Proof. Since $L: V \rightarrow V$ is a symmetric linear map, $V$ splits into the orthogonal sum $V=\operatorname{ker} L+\operatorname{im} L$, and $U=\operatorname{im} L$. Then each $x \in V$ can be written as $x=x_{0}+x_{1}$ with $x_{0} \in \operatorname{ker} L$ and $x_{1} \in U$. Note $L x=L x_{1}=L_{1} x_{1}$ where $L_{1}=$ $L \mid U: U \rightarrow U$ is a nonsingular symmetric linear map. This implies

$$
R x y= \pm L x L y= \pm L_{1} x_{1} L_{1} y_{1}= \pm \Lambda^{2} L_{1}\left(x_{1} y_{1}\right)
$$

As noted in $\S 2$ the space $\Lambda^{2} V$ can be written as $\Lambda^{2} V=\operatorname{ker} R+\operatorname{im} R$, with $\Lambda^{2} U=\operatorname{im} R$ here. Then $x y=\left(x_{0}+x_{1}\right)\left(y_{0}+y_{1}\right)=x_{0} y_{0}+x_{0} y_{1}+x_{1} y_{0}+x_{1} y_{1}$ $=(x y)_{0}+(x y)_{1}$ and $(x y)_{1}=x_{1} y_{1}$. Hence $R x y=R(x y)_{1}=R_{1} x_{1} y_{1}$, where $R_{1}=$ $R \mid \Lambda^{2} U$ is a nonsingular symmetric linear map.

It follows from the above two equations for $R x y$ that $R=\Lambda^{2} L$ if and only if $R_{1}=\Lambda^{2} L_{1}$.

Let $e_{1}, \ldots, e_{n}$ be a basis of $V$ such that $e_{1}, \ldots, e_{k}$ is a basis of $U=\operatorname{im} L$ and $e_{k+1}, \ldots, e_{n}$ is a basis of $\operatorname{ker} L$. Then $R e_{j} e_{j}=R_{1}\left(e_{i} e_{j}\right)$ for $1 \leqslant i, j \leqslant k$ and $R e_{i} e_{p}=R e_{p} e_{q}=0$ for $k+1 \leqslant p, q \leqslant n$. It follows that $\phi(R)=\phi\left(R_{1}\right)$ and $\psi(R)=$ $\psi\left(R_{1}\right)$, since all terms not involving only the matrix of $R_{1}$ must vanish. Therefore $\phi(R)+\frac{1}{4} \psi(R)=\phi\left(R_{1}\right)+\frac{1}{4} \psi\left(R_{1}\right)$, and the conclusion of the theorem follows from Proposition 7.2 applied to $R_{1}: \Lambda^{2} U \rightarrow \Lambda^{2} U$ and $L_{1}: U \rightarrow U$. Q.E.D.

REMARK. The sign in $\pm \Lambda^{2} L$ actually determines two disjoint classes of maps. Namely, if $L, M: V \rightarrow V$ are symmetric linear maps of rank $\geqslant 3$, then $\Lambda^{2} L$ $\neq-\Lambda^{2} M$. This follows from the remark in [9, top of p. 201], via the equation $\Lambda^{2} L(x y)=\Lambda^{2} L_{1}\left(x_{1} y_{1}\right)$ obtained in the proof of Theorem 7.6 above.

The following theorem is the main result of this paper.

THEOREM 7.7. A curvature operator $R: \Lambda^{2} V \rightarrow \Lambda^{2} V$ of rank $>1$ can be factored as $R=\Lambda^{2} L$, for a suitable symmetric linear map $L: V \rightarrow V$, if and only if it satisfies each of the following conditions.

(i) $R$ preserves decomposability.

(ii) $R$ satisfies the Bianchi identity.

(iii) $\phi(R)+\frac{1}{4} \psi(R)>0$. (If $n \equiv 3(\bmod 4)$, then $\operatorname{det}(R \mid \operatorname{im} R)>0$ may be substituted.)

(iv) Either $\Lambda^{2} R \neq 0$ and $R$ is not a correlation if rank $R=6$ or $\Lambda^{2} R \equiv 0$ and rank $R=$ type $R=3$.

In case rank $R \leqslant 1$, the conditions (ii), (iii), and (iv) are deleted. 
Proof. For rank $R>1$, see Theorems 7.3, 7.5, 7.6 and the subsequent Remark. For rank $\leqslant 1$, see Proposition 6.1 and its proof; in this case $\Lambda^{2} R \equiv 0$. Q.E.D.

In conclusion, we make some observations about curvature operators not factorizable as $R=\Lambda^{2} L$. First, if one is willing to concede condition (iii) above, then one should consider the factorization $R= \pm \Lambda^{2} L$. Second, if one wants $R$ to preserve decomposability and have rank $>3$, then Theorem 7.3 and Corollary 7.4 force $\Lambda^{2} R \equiv 0$.

ExAmPLE. Let $e_{1}, \ldots, e_{n}$ be an othonormal basis of $V$ and define (for $i<j$ )

$$
R e_{i} e_{j}= \begin{cases}e_{1} e_{j}, & \text { if } i=1, \\ 0, & \text { otherwise. }\end{cases}
$$

Clearly $\Lambda^{2} R \equiv 0$ and $R$ preserves decomposability. Since rank $R=n-1$, this $R$ is not factorizable as $R= \pm \Lambda^{2} L$ when $n>4$. Note that $U=V$ and that $R$ satisfies the Bianchi identity.

The general nonfactorizable curvature operator does not preserve decomposability. If it satisfies the Bianchi identity, then it has the decomposition $R=\Lambda^{2} L_{1}$ $+\cdots+\Lambda^{2} L_{p}$ mentioned in $\S 1$ (cf. [4, p. 102]).

\section{REFERENCES}

1. E. Bertini, Introduzione alla geometria proiettiva degli iperspazi, 2nd ed., Principato, Messina, 1923.

2. W. L. Chow, On the geometry of algebraic homogeneous spaces, Ann. of Math. (2) 50 (1949), 32-67.

3. J. Dieudonné, La géométrie des groupes classiques, 2nd ed., Springer-Verlag, Berlin, 1963.

4. H. Jacobowitz, Curvature operators on the exterior algebra, Linear and Multilinear Algebra 7 (1979), 93-105.

5. S. Kobayashi and K. Nomizu, Foundations of differential geometry, Volume II, Interscience, New York, 1969.

6. M. Marcus, Finite-dimensional multilinear algebra, Vols. I and II, Dekker, New York, 1974 and 1975.

7. N. A. Rozenson, On Riemannian spaces of class one, Izv. Akad. Nauk SSSR Ser. Mat. 4 (1940), 181-192; 5 (1941), 325-351; 7 (1943), 253-284.

8. T. Y. Thomas, Riemannian spaces of class one and their characterization, Acta Math. 67 (1936), 169-211.

9. J. Vilms, Local isometric imbedding of Riemannian n-manifolds into Euclidean $(n+1)$-space, J. Differential Geometry 12 (1977), 197-202.

10. H. Whitney, Geometric integration theory, Princeton Univ. Press, Princeton, N. J., 1957.

11. N. N. Yanenko, Some questions of the theory of imbeddings of Riemannian metrics into Euclidean spaces, Uspehi Mat. Nauk (N.S.) 8,no. 1 (53),(1953), 21-100.

Department of Mathematics, Colorado State University, Fort Collins, Colorado 80523 